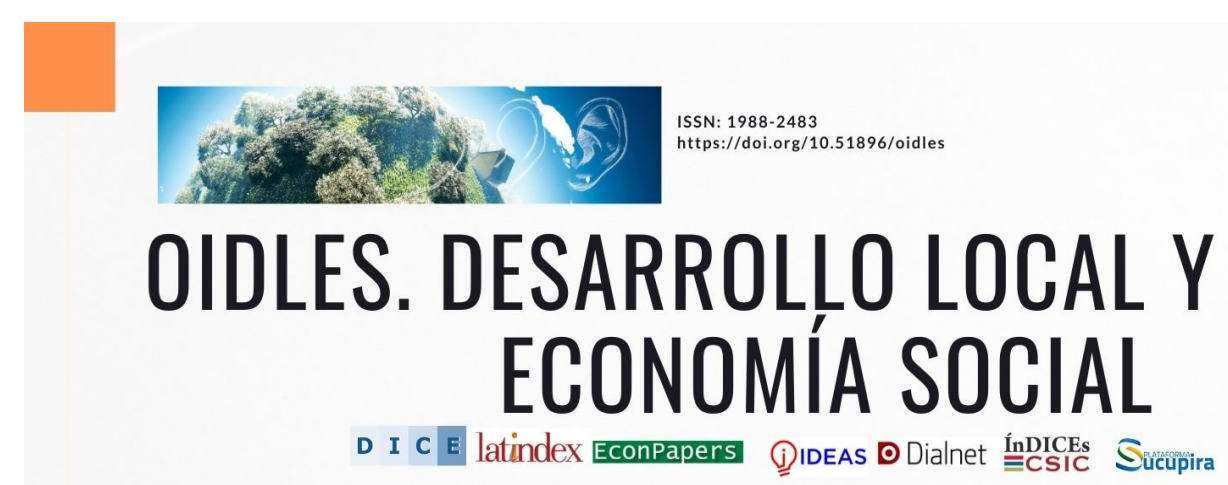

\title{
RESPUESTA DEL CULTIVO DE CEBOLLA PERLA (Allium cepa L.) A LA FERTILIZACIÓN ORGÁNICA, CANTÓN CUMANDÁ, PROVINCIA DE CHIMBORAZO
}

Ing. Mónica Munzón Quintana, MSc'1 Ing. Jonny Barreto Paz ${ }^{2}$

Para citar este artículo puede utilizar el siguiente formato:

Mónica Munzón Quintana y Jonny Barreto Paz: "Respuesta del cultivo de cebolla perla (Allium cepa L.) a la fertilización orgánica, Cantón Cumandá, provincia de Chimborazo", Revista OIDLES, Vol 15 № 30 (junio 2021). En línea:

https://www.eumed.net/es/revistas/oidles/vol-15-no-30-junio-2021/cultivo-cebolla-perla

\section{RESUMEN}

El presente trabajo de investigación experimental se llevó a cabo en la finca de la familia Álvarez Carrión ubicada en el cantón Cumandá provincia de Chimborazo, sobre la respuesta del cultivo de cebolla perla variedad Earli Texas grano 502 donde se utilizó fertilizantes orgánicos foliares y sólidos en varias dosis. Se empleó el diseño de bloques completos al azar (DBCA) con siete tratamientos, un testigo absoluto y uno comercial (tres repeticiones). Para la comparación de las medias se empleó el Test de Tukey al $5 \%$ de probabilidad. Las variables que se evaluaron fueron cinco, entre ellas, rendimiento en kg ha-1. La fertilización orgánica combinada de Eco Abonaza 50\% - FertiEstim plus $50 \%$ dio mejor resultado en todas las variables. El rendimiento fue de $5279 \mathrm{~kg}^{-1 \text { ha }}$, lo que permitió una utilidad de $\$ 433,7$ por hectárea.

Palabras clave: Fertilización orgánica, Fertilización foliar, Eco Abonaza, FertiEstim plus.

\section{RESPONSE OF THE CULTURE OF PEARL ONION (Allium cepa L.) TO ORGANIC FERTILIZATION, CUMANDÁ CANTON, CHIMBORAZO PROVINCE}

\section{ABSTRACT}

The present experimental research work was carried out at the Álvarez Carrión family farm located in the Cumandá canton, Chimborazo province, on the response of the cultivation of pearl onion variety Earli Texas grain 502 where foliar and solid organic fertilizers were used in several dose. The randomized complete block design (DBCA) was used with seven treatments, one absolute control and one commercial (three replications). For the comparison of the means, the Tukey test was used at $5 \%$ probability. The variables that were evaluated were five, among them, yield in $\mathrm{kg}$ ha- 1 . The 
combined organic fertilization of Eco Abonaza $50 \%$ - FertiEstim plus $50 \%$ gave better results in all variables. The yield was $5,279 \mathrm{~kg}$ ha, which allowed a profit of $\$ 433.7$ per hectare.

Keywords: Organic fertilization, Foliar fertilization, Eco Abonaza, FertiEstim plus

\section{INTRODUCCIÓN}

La cebolla perla (Allium cepa $\mathrm{L}$ ) es un producto de gran demanda a nivel mundial siendo esta una de las hortalizas de mayor consumo, gracias a su variedad de utilización para gastronomía internacional. En la región latinoamericana uno de nuestros principales compradores de cebolla perla para el Ecuador es el mercado colombiano.

Es una hortaliza que ocupa el tercer lugar en términos de superficie cosechada a nivel mundial, solo superada por la papa y el tomate. (GOBIERNO DEL ESTADO PLURINACIONAL DE BOLIVIA, 2020)

El género Allium se encuentra constituido por especies cultivadas y ornamentales económicamente significativas. Las principales especies cultivadas son: ajo, puerro, cebolla larga o junca, cebolla de huevo y cebollinos chinos; sin embargo, la tendencia actual que presenta el establecimiento de estos sistemas productivos se encuentra determinada por el uso intensivo de agroquímicos y abonos no estabilizados.Se encuentra conformado por especies vegetales de gran importancia comercial y para la alimentación a nivel nacional y mundial. Especies como A. fistulosum, A. sativum, A. cepa y A. ampeloprasum representan renglones principales de la economía de países como China, Japón y Estados Unidos. (Quintero Herrera, 2016)

La cebolla es originaria de las regiones secas de Irán y el oeste de Pakistán. Según la referencia de algunos botánicos, la misma no se encuentra en estado silvestre. La distribución y desarrollo de la especie ocurrió desde Asia Occidental y países del mediterráneo, hasta América, donde fue introducida por los viajeros conquistadores en el 1492. (Cuases Chingal, 2018)

Es una especie de gran importancia económica, de la cual hay gran demanda en el país. Sin duda, esta hortaliza es uno de los alimentos primordiales y complementarios de la canasta familiar. Las variedades de cebolla son numerosas y presentan bulbos de diversas formas y colores. Es una de las hortalizas más importantes, en el Ecuador se produce, la cebolla larga o de rama y las de bulbo (perla y paiteña) se producen en zonas del litoral y en la sierra; su uso es para la alimentación humana como especias y condimento. (Mólina Patrón, 2020)

La producción de cebolla en el Ecuador fluctúa de 30000 a $50000 \mathrm{Tm}$, provenientes de las provincias más productoras, Chimborazo, Tungurahua, Manabí y Santa Elena; cuyo costo de inversión están en el rango de \$8000 a \$10 000 ha/año, generando utilidades de 700 a 1000 qq/ha, que son ofertados y comercializados dependientes del precio vigente en los mercados locales. (Reyes Carrera, 2018) 
Existen una variedad de abono orgánicos, siendo el compost uno de los de mayor demanda principalmente para la producción de hortalizas como la cebolla perla sin embargo, al igual que los productos químicos, actualmente las empresas distribuidoras de insumos, ofertan varios tipos de compost, cada uno caracterizado por su composición y diferente efecto sobre los cultivos, y es dicha diversidad la que demanda la puesta en marcha de investigaciones encaminadas a la evaluación del desarrollo fenológico del cultivo de cebolla perla con diferentes abonos orgánicos. (Mólina Patrón, 2020)

La presente investigación consistió en determinar la respuesta del cultivo de cebolla perla, a la aplicación de fertilizantes orgánicos en el cantón Cumandá, de la provincia del Chimborazo, en un área experimental de $490 \mathrm{~m}^{2}$ desde el mes de julio a octubre del 2016.

\section{Objetivo}

Evaluar la respuesta del cultivo de cebolla perla (Allium cepa L), a la aplicación de fertilizantes orgánicos en el cantón Cumandá.

\section{METODOLOGÍA}

\section{Localización y características climáticas}

El presente trabajo experimental se lo realizó en el cantón Cumandá, provincia de Chimborazo con las siguientes coordenadas UTM Este 684173; Sur 9732370. La temperatura promedio es de $26^{\circ} \mathrm{C}$, con una precipitación media anual de $2250 \mathrm{~mm}$ y una humedad relativa de $87 \%$, con una Topografía: irregular. De tal manera que su suelo es franco arcilloso, cuenta con una zona de vida de un bosque húmedo tropical. Para la ejecución se utilizó los siguientes materiales: cintas para medir, libreta de campo, bandejas de germinación, sustrato, semilla de cebolla perla, surcadora, Biol, Eco abonaza, estiércol, fertiestim plus, cinta de goteo para riego, uniones, codos, pala, bomba de riego, tanque, bomba de mochila, tuberías, llave de paso.

\section{Características agronómicas de cebolla perla}

Se empleó la variedad de cebolla perla Earli Texas grano 502. Presenta un cuello firme, lo que le confiere muy buena capacidad de almacenamiento, bulbos amarillos y dorados en forma globosa con excelente rendimiento. Se produce en suelos de textura media: francos arcillosos o francos arenosos y el tiempo de cosecha tiene un ciclo intermedio, entre 95-115 días dependiendo de la época de siembra y la zona de cultivo.

\section{Diseño Experimental}

El tipo de investigación que utilizamos en este proyecto es la investigación exploratoria que permitió identificar el efecto favorable, desfavorable o nulo de la utilización la respuesta del cultivo de cebolla perla, a la aplicación de fertilizantes orgánicos de tres fertilizantes foliares. El tipo del diseño experimental que se utilizó es el Diseño de Bloques Completos al Azar (DBCA) con 9 tratamientos y 
3 repeticiones y el número total de parcelas experimentales fue 27 . El esquema de análisis de la varianza se explica en la tabla 1

Se realizó la determinación de la altura de las plantas a los 30, 60 y 90 días. El peso del bulbo se consideró con 30 muestras al azar del área útil de cada parcela. Para la variable Vigor de la planta se apreció visualmente los 75 días después del trasplante, considerando el color y turgencia del follaje. El rendimiento se lo expreso en $\mathrm{Kg} / \mathrm{ha}$ en donde se pesó la producción total del ensayo de cada tratamiento y de cada repetición.

\section{Limite espacial}

Distancia entre plantas: $\quad 0.17 \mathrm{~m}$

Distancia entre hileras: $\quad 0.20 \mathrm{~m}$

Numero de tratamientos: $\quad 9$

Número de repeticiones: $\quad 3$

Área de la parcela: $\quad 12 \mathrm{~m}^{2}(4 \mathrm{~m} \times 3 \mathrm{~m})$

Área útil de la parcela: $\quad 5.36 \mathrm{~m}^{2}(1.80 \mathrm{~m} \times 2.98 \mathrm{~m})$

Área total del ensayo: $\quad 490 \mathrm{~m}^{2}$

Área útil del ensayo: $\quad 144.72 \mathrm{~m}^{2}$

La presente investigación se cumplió mediante los siguientes métodos: Método Deductivo, este método parte de los datos generales como es la respuesta del cultivo de cebolla perla a la fertilización orgánica. Los mismos que fueron aplicados en la parcela experimental. El método Inductivo, parte de datos particulares como es la utilización de la información obtenida en la parcela para general conclusiones a nivel de hectárea.

\section{Análisis estadístico}

Los datos tabulados fueron procesados en el Software SPSS. Se utilizó los estadísticos descriptivos, la Prueba ANOVA de un solo factor, en columna separada para cada nivel de factor, con un $95 \%$ de nivel de confianza, Se presentaron diferencias en los tratamientos por lo que se utilizó la prueba Tukey, con un nivel de significancia del 5\%. El Método Analítico, permitió comprender la respuesta del cultivo de cebolla perla a la fertilización orgánica. Y el Método Sintético, este método permitió comprender las diferentes variables de estudio para agruparlas compararlas y a su vez generar unas conclusiones en forma resumida.

\section{Abonos orgánicos utilizados en la investigación}

\section{Eco Abonaza}

Es un abono compostado, obtenido de la mineralización de diferentes residuos vegetales y animales de granjas certificadas, el cual se convierte en un producto libre de patógenos, con alto contenido de materia orgánica y nutrientes. 
Para la aplicación del Eco Abonaza se recomienda su aplicación en la preparación del suelo antes de pasar la última rastra con la finalidad de incorporarlo en el suelo. Se recomienda aplica a inicio y final del invierno, si cuenta con riego se puede aplicar Eco Abonaza durante todo el año. Presentación de 23 kilogramos.

La composición del abono compostado se indica en la Tabla 2.

\section{Humus}

El humus de lombriz es el resultado de la descomposición de la materia orgánica por acción biológica de las lombrices (roja californiana - Eisenia foetida) y microorganismos que actúan a través del tiempo, ayuda a mejorar la estructura del suelo, acelerar el proceso de humificación, aumentar la capacidad de retención del agua, aumentar la capacidad de intercambio catiónico del suelo, estimular la actividad y desarrollo de los microorganismos, aumentar la eficacia de los abonos minerales acelerando la recuperación de la fertilidad. (Salinas Vásquez, Sepúlveda Morales, \& Sepúlveda Chavera, 2014). La aplicación del producto se recomienda en contacto directo con las raíces o lo más cerca posible de estas. En horticultura se recomienda de 100 a 150 gramos/planta. (Lombec, 2021). Es así como en la Tabla 3 se indica la composición del humus.

\section{FertiEstim plus}

Es una formulación única, un bioestimulante de raíz fabricado en los Estados Unidos exclusivamente para FERTISA por Cytozyme Laboratorios Inc. En su fabricación, se usa un proceso novedoso que combina alta tecnologías de fermentación de extractos de algas marinas con quelatacióncomplejación de minerales. Promueve el crecimiento y desarrollo de las plantas y aumenta la productividad de los cultivos, así como la calidad de sus frutos.

Provee componentes que reducen los efectos del estrés ambiental que, junto a las tasas de bioactividad y el sinergismo con el resto de su formulación, le permite ser un producto altamente eficiente, penetra muy rápidamente en la planta con un bajo riesgo de lavado por la lluvia. (Alvarez Morales, 2018). FertiEstim Plus promueve el crecimiento y desarrollo de las plantas, aumenta la productividad de los cultivos, así como la calidad de sus frutos. (FERTISA , 2021). La ficha técnica del producto se indica en la Tabla 4.

\section{Biol}

El biol, el cual es una alternativa natural, capaz de promover y estimular el desarrollo de las plantas y sobre todo mejora y activa el poder germinativo de las semillas. Este puede ser diseñado y enriquecido en dependencia de las necesidades nutricionales y fisiológicas que requiera el cultivo. Para ello se tiene en cuenta la disponibilidad de la materia prima a utilizar, algunas de las más importantes son el estiércol de animales, restos de alimentos, de cosechas, de podas, entre otras; a las que se les añade otros componentes tales como: agua, melaza, leche y leguminosas. (Pérez Mendéz, Peña Peña, Lago Hechemendia, Batista Yero, \& Hechavarría Hérnandez, 2017) 


\section{Siembra}

La siembra por trasplante se realizó luego de un riego localizado hasta que el suelo quede en capacidad de campo. Para la siembra se utilizó un espeque, depositando una planta de cebolla perla a $17 \mathrm{~cm}$ entre planta y a $20 \mathrm{~cm}$ por hielera; dando un número total de 353 por parcela; a los 8 días se procedió a resembrar.

\section{Fertilización del cultivo de cebolla perla}

Las aplicaciones de los diferentes tratamientos que se realizaron al ensayo se suministraron en dos aplicaciones diferentes; la primera se aplicó todos los fertilizantes orgánicos sólidos, una semana antes del trasplante y la segunda aplicación con los fertilizantes orgánicos líquidos suministrada al ensayo se efectuará a 5 días, 50 días y a 75 días después del trasplante, respectivamente a cada tratamiento.

\section{Diseño Agronómico para la efectividad del riego}

En el ensayo se utilizó un riego por goteo, para lo cual, se estima la precipitación de la lluvia en la zona y la evapotranspiración, mediante datos obtenidos por el INAMHI, se arrollando el diseño agronómico con datos trabajados en el Software Cropwat 8.0 para ser eficaz al momento de aplicar la lámina de agua al trabajo experimental, evitando así un exceso de agua que causará daño al sistema radicular y posteriormente a la planta.

\section{Resultados}

Altura de planta a los 30 días después de la siembra: Según el análisis estadístico de las medidas descriptivas, no existen diferencias significativas para esta variable. Se indica que el tratamiento 1 (EcoAbonazan50\% - Humus $50 \%$ ) tuvo el mayor promedio en la variable altura a 30 días con 26,7 $\mathrm{cm}$, y el tratamiento que muestra el menor promedio corresponde al tratamiento 4 (Eco Abonaza $100 \%$ ) con 17, $6 \mathrm{~cm}$. Según el ANDEVA del análisis de varianza indica que los tratamientos de altura de planta a los 30 días no se encuentran diferencia significativa por lo tanto se muestra que el coeficiente de variación es de $13,86 \%$ Cuadro 1

Altura de planta a los 60 días después de la siembra: La mayor altura promedio a los 60 días corresponde al tratamiento número 3 (Eco Abonaza 50\% - FertiEstim plus $50 \%$ ) con 49,20 cm, la menor altura de la planta es con la aplicación del tratamiento 4 (Eco Abonaza 100\%) con 32,4 cm. Según el ANDEVA del análisis de varianza de la variable altura de la planta a los 60 días indica que No se encontró diferencia significativa por lo tanto se muestra que el coeficiente de variación es de 15,80\%. Cuadro 1

Altura de planta a los 90 días después de la siembra: El análisis estadístico de las medidas descriptivas, indica que el tratamiento que obtuvo el menor promedio es el 6 (Humus 100\%) con 42,8 $\mathrm{cm}$ y el que tuvo el mayor promedio en cuanto a la altura de planta de 90 días fue el tratamiento 3 (Eco Abonazan 50\% - FertiEstim plus $50 \%$ ) con 56,8 cm. Según el ANDEVA del análisis de varianza 
indica que los tratamientos de altura de planta a los 90 días no se encuentran diferencia significativa por lo tanto se muestra que el coeficiente de variación es de 17,36\%. Cuadro 1

Peso del bulbo (g): El análisis estadístico de las medidas descriptivas de la variable peso del bulbo (g) indica que el tratamiento que tiene un promedio bajo es el tratamiento 9 (testigo absoluto) con 19,3 gramos y el tratamiento 3 (EcoAbonazan 50\% - FertiEstim plus $50 \%$ ) presenta el mayor promedio con 53,9 gramos. Según el ANDEVA del análisis de varianza indica que los tratamientos muestran diferencias significativas, el coeficiente de variación es de 6,56\%. Cuadro 2

Rendimiento $\mathrm{Kg} / \mathrm{ha}$

Una vez cosechadas las cebollas, se procedió a realizar el cálculo de rendimiento de los tratamientos en $\mathrm{kg} / \mathrm{ha}$ rendimiento $\mathrm{kg} / \mathrm{ha}$ donde el tratamiento número 3 (Eco Abonaza $50 \%$ FertiEstim plus $50 \%$ ) presenta el mayor promedio $527,9 \mathrm{~kg} / \mathrm{ha}$, el tratamiento que tiene un promedio bajo es el tratamiento número 9 (testigo absoluto) con 188,9. Según el ANDEVA en el análisis de varianza indica que los tratamientos presentan efectos estadísticamente diferentes por lo que es necesaria la aplicación de la prueba de comparación de Tukey al $5 \%$ de probabilidad. Todos los tratamientos presentan medias significativamente diferentes, con un coeficiente de variación de 6,57

\section{$\%$. Cuadro 3}

Análisis económico

En el Cuadro 4 se muestra la relación que hubo entre los rendimientos y costo de producción, y el precio que estaba la cebolla en el mes de noviembre. Siendo el tratamiento comercial que presentó menor perdida, cabe observar que el tratamiento 3 generó mayor ingreso económico, pero en comparación en los costos totales, no se ve ganancias netas para el tiempo transcurrido y los costos de mantenimiento.

\section{DISCUSIÓN}

De acuerdo con la investigación planteada en base al objetivo de realizar un análisis estadístico de la respuesta del cultivo de cebolla perla, a la aplicación de fertilizantes orgánicos.

Mólina Patrón (2020) expresa que los promedios de altura de planta a los 30 días después de la siembra con el compost Ecogreen son de una altura menor con $15.19 \mathrm{~cm}$, lo que concuerda con este autor en vista que el tratamiento 4 (Eco Abonaza 100\%) con 17,6 cm. Según los resultados de Blanco Torres (2017) se aprecia la prueba de comparación de medias Duncan a nivel del 0.05, donde se observa que el tratamiento T3=3 l/mochila de Biol tuvo mayor altura de planta con promedio de $61.63 \mathrm{~cm}$, el cual es estadísticamente superior a los demás tratamientos; seguido de los tratamientos T2=2 l/mochila de Biol y el T1=testigo que con 55.07 y $45.94 \mathrm{~cm}$ respectivamente, lo que guarda relación con los resultados de este autor en donde el mayor promedio en cuanto a la altura de planta de 90 días fue el tratamiento 3 (Eco Abonaza 50\% - FertiEstim plus $50 \%$ ) con 56,8 cm.

Lozano Flores (2017) en su investigación explica que las aplicaciones más crecientes de las dosis de pollaza generaron un efecto respuesta lineal positiva sobre las variables predictoras: Diámetro del cuello de la planta, diámetro del bulbo, longitud de la planta, peso de la planta y rendimiento 
(variables dependientes) y altas relaciones de correlación superiores a los $93 \%$, lo que concuerda con este autor que indica que una vez cosechadas las cebollas, se procedió a realizar el cálculo de rendimiento de los tratamientos en $\mathrm{kg} / \mathrm{ha}$ rendimiento $\mathrm{kg} / \mathrm{ha}$ donde el tratamiento número 3 (Eco Abonaza 50\% - FertiEstim plus $50 \%$ ) presenta el mayor promedio 527,9 kg/ha.

Los resultados obtenidos por Nuñez Tapia (2015) indican que peso promedio de los bulbos, en la evaluación de niveles de fertilización, en la respuesta del cultivo de cebolla perla a tres tipos de abonos orgánicos y tres niveles de fertilización edáfica se observa significancia estadística al $5 \%$ para, abonos, tratamientos, niveles y para la interacción AxN, en tanto que, no se detecta ninguna significancia para factorial vs. testigo y repeticiones. El promedio general fue de $96.26 \mathrm{~g} / \mathrm{bulbo}$, lo que difiere este autor en donde el tratamiento 3 (Eco Abonaza 50\% - FertiEstim plus $50 \%$ ) presenta el mayor promedio con 53,9 g. Según el ANDEVA del análisis de varianza indica que los tratamientos muestran diferencias significativas, el coeficiente de variación es de 6,56\%.

\section{CONCLUSIONES}

Según el análisis e interpretación estadística de los resultados experimentales obtenidos en el presente trabajo de investigación, se concluye lo siguiente:

La mayor altura de planta a los 30, 60 y 90 días después del trasplante lo obtuvo la aplicación (Eco Abonaza 50\% - FertiEstim plus 50\%).

En la variable de peso de bulbo el tratamiento que presento mayor promedio fue el número 3 (Eco Abonaza 50\% - FertiEstim plus 50\%.).

Los tratamientos con aplicación de abonos orgánicos, en sus diferentes dosis, no mostraron resultados favorables debido a las condiciones del clima en el sector donde se realizó la investigación.

\section{Tabla 1}

Tratamientos en estudio

\begin{tabular}{|c|c|c|c|}
\hline TRATAMIENTO & DESCRIPCIÓN & DOSIS/ ha & DOSIS/TRATAMIENTO \\
\hline 1 & Eco Abonaza 50\% - Humus 50\%. & $500 \mathrm{~kg} / 500 \mathrm{~kg}$ & $600 \mathrm{gr} / 600 \mathrm{~g}$ \\
\hline 2 & Eco Abonaza 50\% - Biol 50\%. & $500 \mathrm{~kg} / 1 \mathrm{I}$ & $600 \mathrm{gr} / 12 \mathrm{cc}$ \\
\hline 3 & $\begin{array}{l}\text { Eco Abonaza } 50 \% \text { - FertiEstim plus } \\
50 \% \text {. }\end{array}$ & $500 \mathrm{~kg} / 1 \mathrm{I}$ & $6 \mathrm{~kg} / 12 \mathrm{cc}$ \\
\hline 4 & Eco Abonaza $100 \%$. & $1000 \mathrm{~kg}$ & $1200 \mathrm{~g}$ \\
\hline 5 & Biol $100 \%$. & 21 & $24 \mathrm{cc}$ \\
\hline 6 & Humus $100 \%$. & $1000 \mathrm{~kg}$ & $1200 \mathrm{gr}$ \\
\hline
\end{tabular}


8

Testigo comercial.
FertiEstim plus $100 \%$.

Testigo absoluto
21

$250 \mathrm{~kg} \mathrm{~N}$

150 kg P2 O5

$250 \mathrm{~kg} \mathrm{~K} 2 \mathrm{O}$
$24 \mathrm{cc}$

$0.30 \mathrm{~kg} \mathrm{~N}$

$0.18 \mathrm{~kg} \mathrm{PO} 5$

$0.30 \mathrm{~K} 2 \mathrm{O}$

Tabla 2

Composición de eco abonaza

\begin{tabular}{|c|c|c|c|}
\hline ELEMENTO & & $\%$ & \\
\hline & Materia Orgánica & & 61.52 \\
\hline Nitrógeno & & & 2.73 \\
\hline Fósforo & & 1.75 & \\
\hline Potasio & & 3.63 & \\
\hline & & & 4.42 \\
\hline & Magnesio & & 1.06 \\
\hline & & & 0.02 \\
\hline & ganeso & & 0.07 \\
\hline & & & 0.02 \\
\hline
\end{tabular}

Tabla 3

Composición del humus

\section{CARACTERISTICA/ELEMENTO}

$\mathrm{pH}$

Colonia bacteriana

Ácidos húmicos

Materia Orgánica

Carbono Orgánico

Relación $\mathrm{C} / \mathrm{N}$

Nitrógeno orgánico (N)

Fósforo orgánico $(\mathrm{P})$

Potasio orgánico $(\mathrm{K})$

\section{VALORES}

8,1

miles de millones por gramo de producto

$9 \%$

$40,90 \%$

$20,14 \%$

9,6

$2,31 \%$

$1,46 \%$

$2,37 \%$ 


\section{Tabla 4}

Ficha tecnica del fertiestim plus

Uso/ Aplicación: Foliar, dosis de $0.75-1$ I /ha. $1-3$ aplicaciones

Presentación: Envases de polietileno de 0.5, 1, 5 y 20 I.

Tipo: Fertilizante líquido

Especificaciones Técnicas

Químicas:

$\%$ Concentración:

Nitrógeno Total $(\mathrm{N})$ $7.2 \%$

Boro (B) $0.024 \%$

Fósforo (P2O5) $4.8 \%$

Cobre (Cu) $0.33 \%$

Potasio (K2O) $3.6 \%$

Azufre (S) $1.2 \%$

Cobalto (Co) $0.018 \%$

Hierro (Fe)

$0.43 \%$

Molibdeno (Mo)

$0.0010 \%$

Manganeso (Mn)

$0.36 \%$

Zinc (Zn)

$0.72 \%$

\section{Cuadro 1.-}

Altura de planta (cm) a los 30, 60 y 90 despues del trasplante

\begin{tabular}{|c|c|c|c|c|}
\hline \multirow[t]{2}{*}{ No. } & TRATAMIENTOS & \multicolumn{3}{|c|}{ PROMEDIOS } \\
\hline & & $30 \mathrm{ddt}$ & $60 \mathrm{ddt}$ & $90 \mathrm{ddt}$ \\
\hline 1 & Eco Abonaza 50\% - Humus 50\%. & $26,7 \mathrm{~ns}$ & $47,8 \mathrm{~ns}$ & $48,7 \mathrm{~ns}$ \\
\hline 2 & Eco Abonaza 50\% - Biol 50\%. & $26,0 \mathrm{~ns}$ & $37,8 \mathrm{~ns}$ & $47,1 \mathrm{~ns}$ \\
\hline 3 & $\begin{array}{l}\text { Eco Abonaza } 50 \% \text { - FertiEstim } \\
\text { plus } 50 \% \text {. }\end{array}$ & $26,2 \mathrm{~ns}$ & $49,2 \mathrm{~ns}$ & $56,8 \mathrm{~ns}$ \\
\hline 4 & Eco Abonaza 100\%. & $17,6 \mathrm{~ns}$ & $32,4 \mathrm{~ns}$ & $44,4 \mathrm{~ns}$ \\
\hline 5 & Biol 100\%. & $26,2 \mathrm{~ns}$ & $36,6 \mathrm{~ns}$ & $52,8 \mathrm{~ns}$ \\
\hline 6 & Humus $100 \%$. & $23,0 \mathrm{~ns}$ & $35,6 \mathrm{~ns}$ & $42,8 \mathrm{~ns}$ \\
\hline 7 & FertiEstim plus $100 \%$. & $23,8 \mathrm{~ns}$ & $35,0 \mathrm{~ns}$ & $46,7 \mathrm{~ns}$ \\
\hline 8 & Testigo Comercial $100 \%$ & $26,6 \mathrm{~ns}$ & $47,8 \mathrm{~ns}$ & $52,9 \mathrm{~ns}$ \\
\hline 9 & Testigo Absoluto & $22,5 \mathrm{~ns}$ & $34,4 \mathrm{~ns}$ & $45,3 \mathrm{~ns}$ \\
\hline & Coeficiente de variación & $13,86 \%$ & $15,80 \%$ & $17,36 \%$ \\
\hline
\end{tabular}
ns no significativo

Cuadro de Análisis de la Varianza (SC tipo III) Altura plantas 30 ddp

\begin{tabular}{lccccc} 
F.V. & SC & gl & CM & F & p-valor \\
\hline Modelo. & 213,37 & 10 & 21,34 & 1,88 & 0,1248 \\
TRATAMIENTOS & 212,92 & 8 & 26,62 & 2,35 & 0,0694 \\
REPETICIONES & 0,45 & 2 & 0,22 & 0,02 & 0,9806 \\
Error & 181,25 & 16 & 11,33 & &
\end{tabular}


Medias con una letra común no son significativamente diferentes $(p>0,05)$ Altura plantas $30 \mathrm{ddt}$

\begin{tabular}{lccc}
\multicolumn{1}{c}{ Tratamientos } & Medias & n & E. E \\
\hline 4: Eco Abonaza & 17,63 & 3 & $1,94 \mathrm{~A}$ \\
9: Testigo Absoluto & 22,43 & 3 & $1,94 \mathrm{~A}$ \\
6: Humus & 23,03 & 3 & $1,94 \mathrm{~A}$ \\
7: FertiEstim plus & 23,77 & 3 & $1,94 \mathrm{~A}$ \\
2: Eco Abonaza /Biol & 26,03 & 3 & $1,94 \mathrm{~A}$ \\
5: Biol & 26,17 & 3 & $1,94 \mathrm{~A}$ \\
3: Eco Abonaza-FertiEstim & 26,20 & 3 & $1,94 \mathrm{~A}$ \\
plus & & & \\
8: Testigo Comercial & 26,57 & 3 & $1,94 \mathrm{~A}$ \\
1: EcoAbonaza-humus & 26,73 & 3 & $1,94 \mathrm{~A}$ \\
\hline
\end{tabular}

Medias con una letra común no son significativamente diferentes $(p>0,05)$

Cuadro de Análisis de la Varianza (SC tipo III) altura de planta a los 60 días

\begin{tabular}{lccccc} 
F.V. & SC & gl & CM & F & p-valor \\
\hline Modelo. & 1063,04 & 10 & 106,30 & 2,25 & 0,0716 \\
TRATAMIENTOS & 1061,91 & 8 & 132,74 & 2,81 & 0,0375 \\
REPETICIONES & 1,13 & 2 & 0,56 & 0,01 & 0,9882 \\
Error & 756,21 & 16 & 47,26 & & \\
Total & 1819,25 & 26 & & &
\end{tabular}

Medias con una letra común no son significativamente diferentes $(p>0,05)$ altura de planta a los 60 días

\begin{tabular}{lccc}
\multicolumn{1}{c}{ Tratamientos } & Medias & $\mathbf{n}$ & E. E \\
\hline 4: Eco Abonaza & 32,43 & 3 & $3,97 \mathrm{~A}$ \\
9: Testigo Absoluto & 34,33 & 3 & $3,97 \mathrm{~A}$ \\
7: FertiEstim & 34,97 & 3 & $3,97 \mathrm{~A}$ \\
6: Humus & 35,60 & 3 & $3,97 \mathrm{~A}$ \\
5: Biol & 36,63 & 3 & $3,97 \mathrm{~A}$ \\
2: Eco Abonaza /Biol & 37,80 & 3 & $3,97 \mathrm{~A}$ \\
1: Eco Abonaza-humus & 47,80 & 3 & $3,97 \mathrm{~A}$ \\
8: Testigo Comercial & 47,80 & 3 & $3,97 \mathrm{~A}$ \\
3: Eco Abonaza-FertiEstim & 49,13 & 3 & $3,97 \mathrm{~A}$ \\
plus & & & \\
\hline Medias con una letra común no son significativamente diferentes $(\mathrm{p}>0,05)$ &
\end{tabular}

Medias con una letra común no son significativamente diferentes $(p>0,05)$

Cuadro de Análisis de la Varianza (SC tipo III) altura de planta a los $\mathbf{9 0}$ ddt

\begin{tabular}{lccccc} 
F.V. & SC & gl & CM & F & p-valor \\
\hline Modelo. & 638,23 & 10 & 63,82 & 1,06 & 0,4454 \\
TRATAMIENTOS & 598,53 & 8 & 74,82 & 1,24 & 0,3399 \\
REPETICIONES & 39,70 & 2 & 19,85 & 0,33 & 0,7249 \\
Error & 967,52 & 16 & 60,47 & & \\
Total & 1605,75 & 26 & & &
\end{tabular}

Medias con una letra común no son significativamente diferentes $(p>0,05)$

\begin{tabular}{lccc}
\multicolumn{1}{c}{ Tratamientos } & Medias & n & E. E \\
\hline 6: Humus & 42,77 & 3 & $4,49 \mathrm{~A}$ \\
4: Eco Abonaza & 44,43 & 3 & $4,49 \mathrm{~A}$ \\
9: Testigo Absoluto & 45,33 & 3 & $4,49 \mathrm{~A}$
\end{tabular}




$\begin{array}{llll}\text { 7: FertiEstim plus } & 46,67 & 3 & 4,49 \mathrm{~A} \\ \text { 2: EcoAbonaza /Biol } & 47,13 & 3 & 4,49 \mathrm{~A} \\ \text { 5: Biol } & 52,77 & 3 & 4,49 \mathrm{~A} \\ \text { 8: Testigo Comercial } & 52,87 & 3 & 4,49 \mathrm{~A} \\ \text { 1: Eco Abonaza-humus } & 54,23 & 3 & 4,49 \mathrm{~A} \\ \text { 3: EcoAbonaza-FertiEstim } & 56,83 & 3 & 4,49 \mathrm{~A} \\ \text { plus } & & & \end{array}$

\section{Cuadro 2.}

Peso del bulbo

\begin{tabular}{|c|c|c|c|c|c|c|c|c|}
\hline & & & & \multicolumn{4}{|c|}{ REPETICIONES } & \\
\hline \multicolumn{4}{|c|}{ TRATAMIENTOS } & 1 & II & \multicolumn{3}{|c|}{ III } \\
\hline \multicolumn{4}{|c|}{1 Eco Abonaza 50\% - Humus 50\%. } & 27,8 & 27,6 & \multicolumn{3}{|c|}{29,5} \\
\hline \multicolumn{4}{|c|}{2 Eco Abonaza 50\% - Biol 50\%. } & 29,2 & 29,9 & \multicolumn{3}{|c|}{30,6} \\
\hline \multicolumn{4}{|c|}{3 Eco Abonaza 50\% - FertiEstim 50\%. } & 54,8 & 53,6 & \multicolumn{3}{|c|}{53,1} \\
\hline \multicolumn{4}{|l|}{4 Eco Abonaza 100\%. } & 27,0 & 28,9 & \multicolumn{3}{|c|}{27,1} \\
\hline \multicolumn{4}{|l|}{5 Biol $100 \%$} & 26,4 & \multicolumn{2}{|l|}{25,1} & \multicolumn{2}{|l|}{26,1} \\
\hline \multicolumn{4}{|l|}{6 Humus $100 \%$. } & 35,2 & \multicolumn{2}{|l|}{35,6} & \multicolumn{2}{|l|}{35,2} \\
\hline \multicolumn{4}{|l|}{7 FertiEstim plus $100 \%$} & 29,2 & \multicolumn{2}{|l|}{32,2} & \multicolumn{2}{|l|}{34,8} \\
\hline \multicolumn{4}{|l|}{8 Testigo Comercial 100\%. } & 52,2 & \multicolumn{2}{|l|}{51,4} & \multicolumn{2}{|l|}{54,4} \\
\hline \multicolumn{4}{|l|}{9 Testigo Absoluto } & 17,9 & \multicolumn{2}{|l|}{25,1} & \multicolumn{2}{|l|}{14,8} \\
\hline \multicolumn{9}{|c|}{ Cuadro de Análisis de la Varianza (SC tipo III) } \\
\hline F.V. & SC & & gl & & & $\mathrm{F}$ & & $\mathrm{p}$-valor \\
\hline Modelo. & 3364,77 & & 10 & & & 68,08 & & $<0,0001$ \\
\hline TRATAMIENTOS & 3359,46 & & 8 & & & 84,97 & & $<0,0001$ \\
\hline REPETICIONES & 5,31 & & 2 & & & 0,54 & & 0,5946 \\
\hline Error & 79,08 & & 16 & & & & & \\
\hline Total & 3443,85 & & 26 & & & & & \\
\hline Medias con una letra com & ún no son & significat & vem & ferente & ,05) & & & \\
\hline Tratamientos & & Medias & & & & & & $\mathbf{E}$ \\
\hline T9: Testigo absoluto & & 19,27 & & & & & & $8 \mathrm{~A}$ \\
\hline T5: Biol 100\% & & 25,87 & & & & & & 8 B \\
\hline T4: Eco Abonaza 100\% & & 27,67 & & & & & & 8 B \\
\hline T1: Eco Abonaza 50\%- & & 28,30 & & & & & & 8 B \\
\hline Humus $50 \%$ & & & & & & & & \\
\hline T2: Eco Abonaza 50\%- & & 29,90 & & & & & & $B \mathrm{BC}$ \\
\hline Biol $50 \%$ & & & & & & & & \\
\hline $\mathrm{T}: 7$ FertiEstim plus $100 \%$ & & 32,07 & & & & & & $B C$ \\
\hline T6: Humus $100 \%$ & & 35,33 & & & & & & $3 \mathrm{C}$ \\
\hline T8: Testigo comercial & & 52,67 & & & & & & $3 \mathrm{D}$ \\
\hline T3: Eco Abonaza 50\%- & & 53,83 & & & & & & $B D$ \\
\hline FertiEstim plus $50 \%$ & & & & & & & & \\
\hline
\end{tabular}

Medias con una letra común no son significativamente diferentes $(p>0,05)$ 
Cuadro 3.

Rendimiento ( $\mathrm{kg} / \mathrm{ha})$

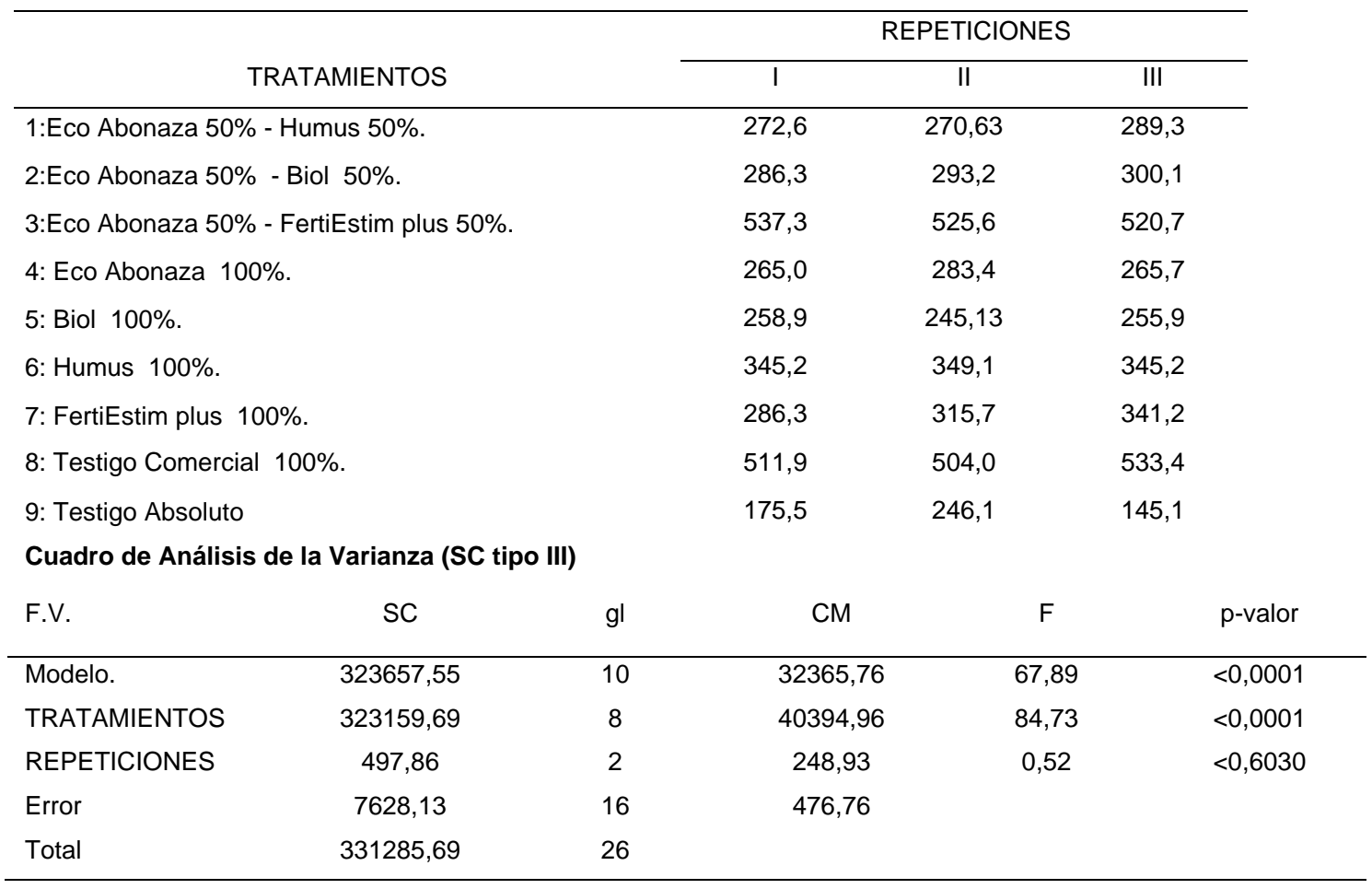

Promedios de la variable rendimiento; $\mathrm{n}=$ número de repeticiones; $\mathrm{E}$. $\mathrm{E}=$ Error experimental

\begin{tabular}{lccc}
\multicolumn{1}{c}{ Tratamientos } & Medias & $\mathbf{n}$ & E. E \\
\hline T9: Testigo absoluto & 1889 & 3 & $12,61 \mathrm{~A}$ \\
T5: Biol 100\% & 2533 & 3 & $12,61 \mathrm{~B}$ \\
T4: Eco Abonaza 100\% & 2713 & 3 & $12,61 \mathrm{~B}$ \\
T1: Eco Abonaza 50\%- & 2775 & 3 & $12,61 \mathrm{~B}$ \\
Humus 50\% & & & \\
T2: Eco Abonaza 50\%- & 2932 & 3 & $12,61 \mathrm{BC}$ \\
Biol 50\% & & & \\
T7: FertiEstim plus 100\% & 3144 & 3 & $12,61 \mathrm{BC}$ \\
T6: Humus 100\% & 3465 & 3 & $12,61 \mathrm{C}$ \\
T8: Testigo comercial & 5164 & 3 & $12,61 \mathrm{D}$ \\
T3: Eco Abonaza 50\%- & 5278 & 3 & $12,61 \mathrm{D}$ \\
FertiEstim plus 50\% & & & \\
\hline
\end{tabular}

\section{Cuadro 4.}

Tabla de valores económicos

\begin{tabular}{clllllll}
\hline TRATAMIENTO & $\begin{array}{l}\mathrm{Kg} / \\
\mathrm{Ha}\end{array}$ & $\begin{array}{l}\text { Costo por } \\
\text { kilogramo }\end{array}$ & $\begin{array}{l}\text { Costo por } \\
\text { tratamiento }\end{array}$ & $\begin{array}{l}\text { Ingreso } \\
\text { total }\end{array}$ & $\begin{array}{l}\text { beneficio } \\
\text { neto }\end{array}$ & \multicolumn{2}{r}{ relación B/C } \\
\hline $\mathbf{1}$ & 2775 & 0.3 & 1200 & 832.5 & $\$ 368$ & $\$ 0.30$ & $\mathrm{X}<1$ \\
$\mathbf{2}$ & 2932 & 0.3 & 1050 & 879.6 & 170.4 & 0.1 & $X<1$ \\
$\mathbf{3}$ & 5279 & 0.3 & 1150 & 1583.7 & 433.7 & 0.4 & $X<1$ \\
\hline
\end{tabular}




\begin{tabular}{lllllllll}
\hline $\mathbf{4}$ & 2714 & 0.3 & 1100 & 814.2 & 285.8 & 0.3 & $X<1$ \\
$\mathbf{5}$ & 2533 & 0.3 & 950 & 759.9 & 190.1 & 0.2 & $\mathrm{X}<1$ \\
$\mathbf{6}$ & 3465 & 0.3 & 1150 & 1039.5 & 110.5 & 0.1 & $\mathrm{X}<1$ \\
$\mathbf{7}$ & 3144 & 0.3 & 1100 & 943.2 & 156.8 & 0.1 & $\mathrm{X}<1$ \\
$\mathbf{8}$ & 5164 & 0.3 & 1300 & 1549.2 & 249.2 & 0.2 & $\mathrm{X}<1$ \\
$\mathbf{9}$ & 1889 & 0.3 & 600 & 566.7 & 33.3 & 0.05 & $X<1$ \\
\hline
\end{tabular}

\section{BIBLIOGRAFÍA}

Alvarez Morales, Y. (2018). El cultivo de pepino (Cucumis sativus, L.), y su comportamiento agronómico por la aplicación de bioestimulantes orgánicos en la zona de Vinces-Ecuador. Tesis. Vinces, Los Rios, Ecuador: Universidad de Guayaquil.

Blanco Torres, E. (2017). Efecto de tres dosis de biol en el cultivo de cebolla (Allium cepa L.) en el centro de investigación y producción Camacani. Tesis. Puno, Perú: Universidad Nacional del Altiplano/Facultad de Ciencias Agrarias.

Cuases Chingal, A. (2018). Estado actual del cultivo de la cebolla blanca (Allium cepa L.) en la zona de. El Angel, Carchi, Ecuador: Universidad Técnica de Babahoyo/Facultad de Ciencias Agropecuarias .

FERTISA . (8 de mayo de 2021). Obtenido de https://fertisa.com/wpcontent/uploads/2019/12/1130868.pdf

GOBIERNO DEL ESTADO PLURINACIONAL DE BOLIVIA. (2020). Informe estadistico de la cebolla. Dirección General de Análisis Productivo. Bolivia: Ministerio de Desarrollo Productivo y Economia Plural.

LOMBEC. (8 de mayo de 2021). Obtenido de https://www.lombec.com/producto_humus_de_lombriz.html

Lozano Flores, C. (2017). Evaluación de dosis de materia orgánica (Pollaza) en el cultivo de cebollita china (Var. Roja chiclayana), bajo condiciones agroecológicas en la provincia de Lamas. Tesis. Tarapoto, Perú: Universidad Nacional de San Martin-Tarapoto/Facultad de Ciencias Agrarias.

Mólina Patrón, E. (2020). Evaluación del efecto de tres tipos de compost en el desarrollo fenológico del cultivo de cebolla perla (Allium cepa L.) en el cantón Mocache, Los Ríos. Tesis de grado. Quevedo, Los Rios, Ecuador: Universidad Técnica Estatal de Quevedo.

Nuñez Tapia, M. (2015). Respuesta del cultivo de cebolla colorada (Allium cepa L.) a tres abonos orgánicos y tres niveles de fertilización edáfica. Tesis. Quito, Ecuador: Universidad Central del Ecuador/Facultad de Ciencias Agricolas/Carrera de Ingenieria Agronómica.

Pérez Mendéz, M., Peña Peña, E., Lago Hechemendia, S., Batista Yero, Y., \& Hechavarría Hérnandez, A. (2017). producción de biol y determinación de sus caracteristicas fisicoquimicos. DIALNET(48).

Quintero Herrera, M. (2016). Caracterización genética de especies pertenecientes al género Allium, de importancia comercial, distribuidas en los departamentos de Risaralda, Antioquia, 
Quindío, Huila, Boyacá y Cundinamarca-Tesis de Maestria en Biologia Vegetal. Pereira: Universidad Tecnológica de Pereira/Universidad del Quindio/Universidad de Caldas.

Reyes Carrera, D. (2018). Análisis económico de la producción de cebolla colorada (Allium cepa I.) en el centro de prácticas Manglaralto, provincia de Santa Elena. Tesis. La Libertad, Santa Elena, Ecuador: Universidad Estatal Peninsula de Santa Elena/Facultad de Ciencias Agrarias.

Salinas Vásquez, F., Sepúlveda Morales, L., \& Sepúlveda Chavera, G. (2014). Evaluación de la calidad quimica del humus de lombriz roja californiana (Eisenia foetida) elaborado a partir de cuatro sustratos órganicos en Arica. Tesis. IDESIA, 32(2), 95-99.

doi:http://dx.doi.org/10.4067/S0718-34292014000200013 